\title{
DIGITALCOMMONS
}

\section{The Importance Of Fortran In The 21st Century}

Walt Brainerd

Follow this and additional works at: http://digitalcommons.wayne.edu/jmasm

Part of the Applied Statistics Commons, Social and Behavioral Sciences Commons, and the Statistical Theory Commons

\section{Recommended Citation}

Brainerd, Walt (2003) "The Importance Of Fortran In The 21st Century," Journal of Modern Applied Statistical Methods: Vol. 2 : Iss. 1 , Article 3.

DOI: $10.22237 /$ jmasm/1051747380

Available at: http://digitalcommons.wayne.edu/jmasm/vol2/iss1/3

This Invited Article is brought to you for free and open access by the Open Access Journals at DigitalCommons@WayneState. It has been accepted for inclusion in Journal of Modern Applied Statistical Methods by an authorized editor of DigitalCommons@WayneState. 


\title{
The Importance Of Fortran In The $21^{\text {st }}$ Century
}

\author{
Walt Brainerd \\ The Fortran Company \\ Tucson, AZ
}

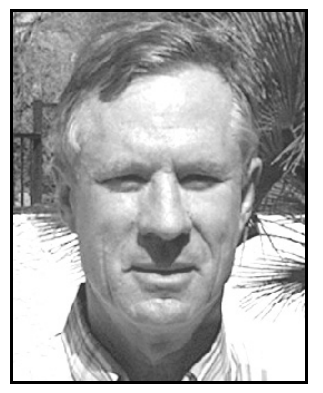

A brief discussion on the history and purpose of Fortran for scientific and engineering computing is given. This leads to the role Fortran, in its various environments, will likely play well into the $21^{\text {st }}$ century.

Key words: Fortran 95, Fortran 2000, F, high performance computing.

\section{Introduction}

Let us start with a bold assertion: Fortran is still the best programming language for numerical/scientific computing. The reasons could be discussed and debated extensively, but they include:

- There is a large investment in scientific software written in Fortran, including extensive libraries.

- There is a large investment in the training and experience of scientists that do programming.

- The language is more straightforward to learn and use than most "modern" languages.

- Fortran produces efficient code.

Walt Brainerd holds one of the first $\mathrm{PhDs}$ in computer science awarded in the USA. He was a leader in the development of Fortran 90 and the co-author of several books, including The Fortran 95 Handbook. He is one of the originators of the $\mathrm{F}$ programming language and maintains the $F$ compiler. Email him atwalt@fortran.com.
- Fortran is very portable: source code compiles on many platforms with little need for conditional compilation and results are consistent, particularly when executed on standard floating point hardware.

The reason I make this statement is because it means that the continued development and implementation of Fortran will be important in the twenty-first century for the same reasons (listed above) that it has been important in the twentieth century.

However, the computing environment is continually changing. What is being done to ensure that Fortran will remain an outstanding tool for scientists and engineers? That is what will be discussed in the remainder of this article.

Language Development and Standardization

In the last quarter century, most of the innovation in the Fortran programming language has come through the group responsible for its standardization. This work is done by the American standards committee J3 under the direction of the International Standards Organization committee WG5. Their web site is: http://www.nag.co.uk/sc22wg5. 
This work continues, and the next standard, being called Fortran 2003 informally is in the process of approval and publication. (See ftp://ftp.j3-fortran.org/j3/doc/standing/007). The major new features include help with interoperation with $\mathrm{C}$ programs and enhanced object-oriented facilities, including inheritance and polymorphism, which enhance the excellent data abstraction features of Fortran 95.

\section{Modern Development Environments}

One of the significant changes for Fortran programmers in recent years has been the availability of modern graphical user interfaces for editing, compiling, executing, and analyzing Fortran programs. Many Fortran implementations include such an environment, in addition to traditional command-line execution and tools.

Unfortunately, these environments are different for almost every compiler. There is hope that there will be an open tool for at least Linux/Unix environments and there does seem to be some convergence by the vendors of Windows compilers to the Microsoft Visual Studio .NET environment. The url: http://msdn.microsoft.com/ vstudio/productinfo/overview/default.asp.

\section{High Performance Computing}

In recent years, several tools have been made available to Fortran (and other programming language) programmers to assist them to take advantage of special high performance computer architectures, such as vector processors, distributed memory multiprocessors, and shared memory multiprocessors. These tools include High Performance Fortran (HPF), OpenMP, and MPI. It is reasonable to expect that these tools will continue to be developed as the new versions of Fortran are implemented.

Free and Open Source Compilers

Unfortunately, due to the smaller number of compilers a vendor may expect to sell, spreading the development costs means that Fortran compilers are moderately expensive. The only open source Fortran compilers are g77 (but unless you need to compile only legacy codes, who wants to use the quarter-century-old version of Fortran?), and Open64, (http://open64.sourceforge.net), a compiler that works only on the Itanium architecture under
Linux. Intel's Linux compiler (http://www.intel.com/software/products/compiler s/index.htm) is available for free, but only for noncommercial use.

There is a g95 project under way to develop a GNU Fortran 95 compiler (http://g95.sourceforge.net). It will probably not be available until after Fortran 2003 compilers come out, so it will again be one step behind. Most vendors of Fortran compilers offer academic discounts.

\section{F}

$\mathrm{F}$ is a subset of Fortran 95 consisting of its modern features and excluding the error-prone older features (http://www.fortran.com/F). Numerical Algorithms Group (the originators of the first Fortran 90 compiler) has made their compiler technology available for this software, which is maintained by the Fortran Company. It is free, but it is not open source.

It is a compiler that can be used to develop production software, because anything that is compiled by the F compiler will also be compiled by any Fortran 95 compiler. It also provides free software for use by academic institutions that want to expose their students to Fortran programming.

\section{Conclusion}

The Fortran language, Fortran compilers, Fortran environments, and Fortran tools continue to advance, along with other computing environments. It looks like Fortran will still be the premier programming language to be used in the twenty-first century when serious numerical and scientific computing needs to be done. Additional information about Fortran can be found at http://www.fortran.com. 